\title{
Changes in Denture Retention With Denture Adhesives and Oral Moisturizers for the Oral Cavity
}

Naoya Ikemura ( $\square$ naota@dent.showa-u.ac.jp )

Showa University School of Dentistry

Yuji Sato

Showa University School of Dentistry

Junichi Furuya

Showa University School of Dentistry

Osamu Shimodaira

Showa University School of Dentistry

Kana Takeda

Showa University School of Dentistry

Takuya Kakuta

Showa University School of Dentistry

Kunihito Yamane

Showa University School of Dentistry

Noboru Kitagawa

Showa University School of Dentistry

\section{Research Article}

Keywords: denture adhesive, denture, oral moisturizer, meals, laboratory research.

Posted Date: April 19th, 2021

DOl: https://doi.org/10.21203/rs.3.rs-395037/v1

License: (c) (1) This work is licensed under a Creative Commons Attribution 4.0 International License.

Read Full License 
1 Changes in denture retention with denture adhesives and oral moisturizers for the oral

2 cavity

3 Naoya Ikemura $^{1}$, Yuji Sato ${ }^{1}$, Junichi Furuya ${ }^{1}$, Osamu Shimodaira ${ }^{1}$, Kana Takeda ${ }^{1}$, Takuya

4 Kakuta $^{1}$, Kunihito Yamane ${ }^{1}$, Noboru Kitagawa ${ }^{1}$

5

$6{ }^{1}$ Department of Geriatric Dentistry, Showa University School of Dentistry Ota Ward,

$7 \quad$ Tokyo Japan

8 Corresponding author: Naoya Ikemura ${ }^{1}$, DDS

9

10

11

12

13

14

15

16

17

18 
Abstract

Background: It is difficult to maintain dentures during meals. This study aimed to assess moisturizers in an oral cavity model.

24 Methods: The following test samples were applied between the palatal plate and the

25 edentulous jaw ridge model: denture adhesive, denture adhesive for dry mouth, oral 26 moisturizer, and denture moisturizer. The retentive force was measured under two

27 conditions: at rest while immersed in water and during function with a $2.5-\mathrm{kg}$ load applied.

28 The plate was pulled perpendicular to the occlusal plane and the retentive force was

29 measured using a digital force gauge.

30 Results: Under dry conditions, denture adhesive for dry mouth and oral moisturizer had

31 a significantly higher retentive force than denture adhesive and denture moisturizer. After

3230 min of immersion in water, the retentive force of the denture adhesive increased while

33 that of the oral moisturizer decreased. After 30 mins of function, the retentive force of the

34 denture adhesive and denture adhesive for dry mouth remained high, while that of the oral moisturizer and denture moisturizer significantly decreased. Between rest and

36 function, the retentive force of the denture adhesive and denture adhesive for dry mouth 
37 was high, and that of the oral moisturizer was low.

38 Conclusions: Immediately after use, denture adhesive for dry mouth exhibited high

39 retentive force, but retention gradually decreased due to its water content.

40 Clinical Significance: Denture adhesives for dry mouth can be useful for retaining 41 dentures during 30-min meals.

42

43

44 Keywords: denture adhesive, denture, oral moisturizer, meals, laboratory research.

45

46

47

48

49

50

51

52

53

54 
Background

In Japan, the elderly population aged 65 years and older is increasing at an unprecedented rate, accounting for $28.4 \%$ of the total population (2019: Ministry of Internal Affairs and Communications). This is the highest number in history and is expected to further increase for the next 30 years. 1 This super-aging population is also important in dentistry. The number of remaining teeth increase as dental treatment progresses. Due to an

61 increased life span, of the number of patients requiring complete dentures is increasing, and complete prosthodontic treatment is expected to become difficult for various reasons. In particular, as aging progresses, it is often difficult to maintain complete dentures due to systemic and oral factors, such as multiple systemic diseases,2,3 oral movement disorders caused by diseases, 4,5 progression of dry mouth caused by side effects of drugs, 6,7 and ridge resorption and mandibular changes caused by aging.8,9 For elderly patients with an aggravated edentulous jaw, denture adhesives are recommended for denture retention and stability during function.10 However, denture adhesives are difficult to remove from the oral mucosa after use. Furthermore, residual denture adhesives are likely to become a breeding ground for oral bacteria, causing aspiration pneumonia.11,12 Dry mouth, which is common among elderly people, is expected to exacerbate the disadvantages of using denture adhesives. Therefore, for some cases, 
73 instead of denture adhesives, denture adhesives for dry mouth or oral moisturizers, which

74 have good cleanability and moisturizing properties are recommended.13,14,15 Ohno et

75 al. measured the retentive force of test samples after drying the plate and discovered that

76 denture adhesives for dry mouth and oral moisturizers had a significantly higher retentive

77 force than denture adhesives. 16

78 In the preliminary experiment, the retentive force of the palatal plate was measured under

79 two conditions: dried and soaked in water for one min. The results suggested that denture

80 adhesives for dry mouth and oral moisturizer can have a higher short-term retentive force

81 than denture adhesives. However, clinically, the retentive force of dentures must be at

82 least $30 \mathrm{~min}$ for eating. Therefore, long-term studies are necessary to further clarify the

83 retentive force of dentures. There have been no reports on changes in denture retention

84 over time with denture adhesives, denture stabilizers for dry mouth, and oral moisturizers.

85 Therefore, the purpose of this study was to recreate the oral cavity during rest and function

86 on a model and elucidate the changes in denture retention over time.

88 Methods

89 I. Test Samples

90 Following a previous study,13 in this study, the test sample was placed between a model 
91 of the edentulous jaw ridge and the denture, and the retentive force of the denture was

92 measured. The following four test samples were assessed: cream-type denture adhesive

93 (NP; New Poligrip ${ }^{\circledR}$ Sa; Glaxo Smith Kline, Tokyo, Japan), gel-type denture adhesive for

94 dry mouth (DM; Pitatto Kaiteki Gel®; NISHIKA), gel-type oral moisturizer (BT; Biotene

95 Oral balance Jell ${ }^{\circledR}$; T\&K, Tokyo, Japan), and cream-type denture moisturizer (DW;

96 Denture Wet ${ }^{\circledR} ;$ DENTCARE) (Figure 1).

97 Characteristics of the test samples

98 NP contain $\mathrm{Na} / \mathrm{Ca} /$ methoxyethylene maleic anhydride copolymer salt. It contains white

99 vaseline as a moisturizing ingredient, and exhibits strong adhesive properties when

100 moistened. DM contains sodium polyacrylate, a sticky substance, and sodium hyaluronate,

101 a water-retaining ingredient. BT contains hydroxyethyl cellulose, an adhesive, and

102 glycerin, a moisturizing agent. DW contains dextrin palmitate, a sticky substance, and

103 squalane, a moisturizing ingredient

104

105 II. Palatal plate manufacturing

106 An impression was made of a silicone edentulous jaw ridge model (G10FE-402K;

107 NISSIN) (Figure 2), and a palatal plate was created using a room-temperature

108 polymerized resin in the form of an occlusal plate to model a denture. Regular relining 
109 (Tokuyama Rebase II; Tokuyama Dental) was performed in order to correct the

110 polymerization shrinkage of the palatal plate. Further, a $0.9-\mathrm{mm}$ traction ring made of Co-

111 Cr was attached to the center of the palate (Figure 3).

113 III. Retentive Force Measuring Device

114 A digital force gauge (Digital Force gauge RX Series ${ }^{\circledR}$; AIKOHENGINEERING, Tokyo,

115 Japan) was used to measure the retentive force, with a hook-shaped traction device 116 attached (Figure 4).

118 IV. Measurement of Retentive Force in Palatal Plate

119 Approximately $2.5 \mathrm{~g}$ of the test sample was applied between the palatal plate and the

120 model so that the sample would cover the entire area by pressure welding. The

121 overflowing test sample was removed and the palatal plate was pressed against the model

122 according to previous studies. $13 \mathrm{~A}$ vertical load was applied for $10 \mathrm{~s}$ using a $2.5-\mathrm{kg}$ disk

123 weight, and the model was then immersed in water. In the experiment, the room

124 temperature was set to $27.0^{\circ} \mathrm{C}$. Following this, the model was removed from the water, a

$125 \quad 2.5-\mathrm{kg}$ load was applied, and the plate was pulled at a speed of $1.0 \mathrm{~N} / \mathrm{s}$ to the direction

126 perpendicular to the occlusal plane using a digital force gauge. The force at which the 
127 palatal plate detached from the model was measured as the retentive force. Four

128 measurements were taken, and the second to fourth measurements were averaged. The

129 series of measurements was repeated three times, and the average value was used as the 130 representative value.

\section{V. Experimental Conditions}

133 The retentive force was measured under two conditions: at rest while immersed in water

134 and during function with load equivalent to the occlusal pressure applied. The experiment

135 was conducted using the four test samples.

136 We applied $2.5 \mathrm{~g}$ of the test sample to the palatal plate, pressed against the model. The

137 load was applied, the plate was pulled, and the retentive force was measured. After

138 pressing the model again and applying a load, the model was immersed in water. After 30

$139 \mathrm{~min}$, it was removed from the water. The load was then applied, the plate was pulled, and

140 the retentive force was measured. This was repeated every $30 \mathrm{~min}$, and after $180 \mathrm{~min}$,

141 measurements were taken every $60 \mathrm{~min}$. The retentive force at $300 \mathrm{~min}$ was considered

142 as the change over time at rest (Figure 5). Further, in order to measure the change over

143 time during function, while submerged in water, a $2.5-\mathrm{kg}$ load was applied five times in

$14410 \mathrm{~s}$ and the model was left for $20 \mathrm{~s}$. Load was applied another five times for $10 \mathrm{~s}$, and 
145 the model was left for $20 \mathrm{~s}$. This process was repeated, and the model was removed from

146 water every $10 \mathrm{~min}$, and load traction was performed. The retentive force at $30 \mathrm{~min}$ was

147 measured and was considered as the change over time during function. (Figure 6)

148

149 VI. Statistical Analysis

150 For statistical analysis, IBM SPSS Statistics 27.0 (IBM) was used, one-way ANOVA was

151 performed, and Tukey's method was used for multiple comparisons. The significance

152 level was set at $5 \%$.

153

154 Results

155 I. Change over time in the retentive force at rest

156 Before immersion, the retentive force of DM and BT was significantly higher than that

157 of NP and DW ( $<0.05)$. However, after $30 \mathrm{~min}$ of immersion in water, the retentive

158 force of NP increased while that of BT decreased. After $60 \mathrm{~min}$, the retentive force of NP

159 and DM was significantly higher than that of BT and DW $(\mathrm{p}<0.05)$. At $300 \mathrm{~min}$, the

160 retentive force of DM, BT, and DW decreased while that of NP remained high (Figure 7).

162 II. Changes over time in retentive force during function 
163 During function, the retentive force of DM and BT before immersion was significantly

164 lower than that of NP and DW $(\mathrm{p}<0.05)$. However, after $10 \mathrm{~min}$ of immersion in water,

165 the retentive force of NP significantly increased $(\mathrm{p}<0.05)$ while that of BT rapidly

166 decreased $(\mathrm{p}<0.05)$. After $30 \mathrm{~min}$, the retentive force of BT and DW significantly

167 decreased. The retentive force of DM showed a slight decreasing trend, but remained high

168 along with the retentive force of NP (Figure 8).

169

170 III. Comparison of changes over time at rest and during function

171 When comparing the changes over time between rest and during function, the retentive

172 force after 30 min during function showed the same tendency as that after 90 min of rest.

173 The change over time in the retentive force during function was approximately three times

174 higher than that at rest. The retentive force of NP and DM was high, and the retentive

175 force of BT and DW was low (Figure 9).

177 Discussion

$178 \quad$ I. Test Samples

179 The amount of test sample placed between the model and the experimental bed was

180 determined based on the results of preliminary experiments and past reports. In other 
181 words, when the entire surface of the model was sufficiently filled and the palatal plate

182 was pressed, the amount of test sample overflowing from the periphery of the palatal plate

183 was already determined. Yamagaki et al. reported that when the entire model is filled with

184 the test sample, the retentive force can be stably measured. In this study, stable

185 measurements were achieved, similar to the preliminary experiment. 13

186 Furthermore, four types of test samples were assessed (NP, DM, highly viscous BT, and

187 DW for different purposes), which can be used as alternatives with reference to previous

188 studies.

189

190 II. Changes over time in retentive force at rest.

191 Under dry conditions, DM and BT, which contain moisturizing ingredients, had a high

192 retentive force. We believe that a certain degree of moisture is necessary to maintain the

193 retentive force of dentures.17 Although NP do not have moisturizing properties, it exerts

194 its retentive force by containing water. Its retentive force increased with time immersed

195 in water. Since BT is fluid, it flows into the water as the immersion time increases, causing

196 its retentive power to decrease. However, since DM also have ingredients that exert

197 retentive force by containing water, its retentive force was high to some extent.16 Further,

198 DW had a lower viscosity than other test samples, which may contribute to its low 
retentive force.

201 III. Changes over time in retentive force during function

202 When assessing the change in the retentive force over time, after $10 \mathrm{~min}$, the increase in

203 the retentive force of NP was the highest. The retentive force of BT decreased sharply.

204 After $30 \mathrm{~min}$, the retentive force of BT and DW was low, and the retentive force of NP

205 slightly decreased. However, NP and DM had a high retentive force. We believe that the

206 repeated application of the load caused a horizontal shift in the palatal plate, and the test

207 sample between the palatal plate and the model was affected by water at an accelerated

208 rate, causing a faster change over time in the retentive force.

210 IV. Comparison of changes over time at rest and during function

211 The change over time in the retentive force during function was approximately three

212 times faster than that at rest. We believe that the repeated application of the load caused

213 a horizontal shift in the palatal plate, and the test sample between the palatal plate and the

214 model was affected by water at an accelerated rate, causing a sudden faster change over

215 time in the retentive force during function. Assuming this occurs in the oral cavity, we

216 believe that there is a considerable difference in the retentive force over time between rest 
217 and during eating and drinking.

218 Assuming an eating and drinking time of $60 \mathrm{~min}$ or longer, NP has a better retentive force.

219 However, assuming general eating and drinking time, DM, which has good cleanability

220 and moisturizing properties, can be useful for retaining dentures.

222 V. Future Outlook

223 In an actual oral cavity, the ridge morphology and the elasticity and thickness of the

224 mucosa differ, which affects the retentive force of the denture.15,18 Therefore, we would

225 like to measure the retentive force of the denture in the oral cavity of patients with

226 complete dentures and clarify the relationship between different conditions (at rest and

227 during function) and the changes in retentive force over time. Further, we would like to

228 clarify the relationship between the degree of dry mouth and the retentive force of

229 dentures as well as the relationship between the objective retentive force evaluation and

230 the satisfaction of subjects for each test sample.

232 Conclusion

233 Immediately after use, DM had high compatibility and exhibited retentive force, but

234 retention gradually decreased due to its water content. On the contrary, immediately after 
235 use, the denture adhesive had low compatibility and exhibited low retentive force, but

236 retention increased due to its water content. These findings suggest that denture adhesives

237 for dry mouth, which have moisturizing properties, have a high retentive force for 30-

238 minute meals.

239

240 Clinical Significance

241

242 List of abbreviations:

243 NP: cream-type denture adhesive

244 DM: gel-type denture adhesive for dry mouth

245 BT: gel-type oral moisturizer

246 DW: cream-type denture moisturizer

247

248 Declarations

249 Ethics approval and consent to participate

$250 \quad$ Consent for publication

$251 \quad$ Availability of data and materials 
256 Declarations

258 Ethics approval and consent to participate: Not applicable

260 Consent for publication: Not applicable

262 Availability of data and material: The datasets used and/or analysed during the current study are available from the corresponding author on reasonable request.

265 Competing interests: The authors declare that they have no competing interests

267 Funding: This study was supported by a Grant-in-Aid for Scientific Research from the 268 Ministry of Education, Culture, Sports, Science, and Technology (Showa University 
269 Grant-in-Aid for Scientific Research (B)) (Grant Number 16K20512).

270

271 Authors' contributions: NI drafted the manuscript. YS contributed advice regarding the

272 manuscript. All authors read and approved the final manuscript.

273

274 Acknowledgments

275 We would like to express our sincere gratitude to the instructors at the Department of

276 Geriatric Dentistry of the School of Dentistry for their cooperation.

277 A portion of the abstract of this paper was presented at the 31st Academic Conference of

278 the Japanese Society of Gerodontology (November 7-8, 2020, in the journal).

279 We would like to thank Editage (www.editage.com) for their writing support of the

280 Abstract.

281

282 Figure Legends

283 figure_1 Test Samples 


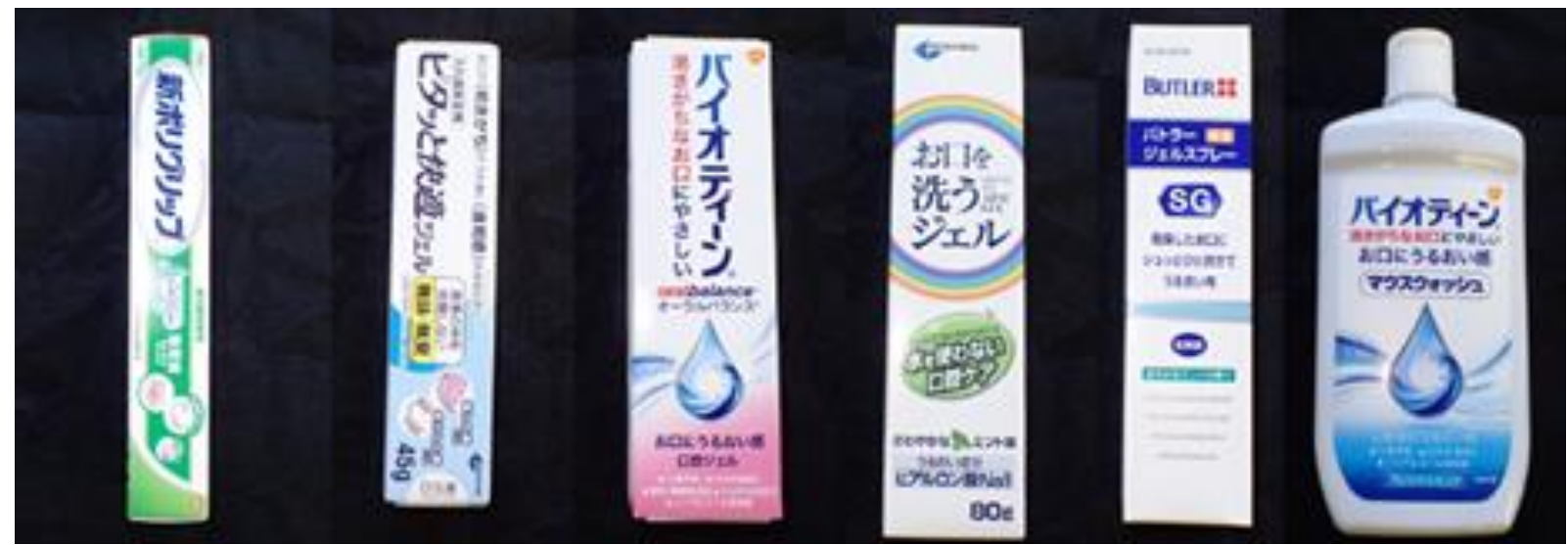

285

286 figure_2 silicone edentulous jaw ridge model

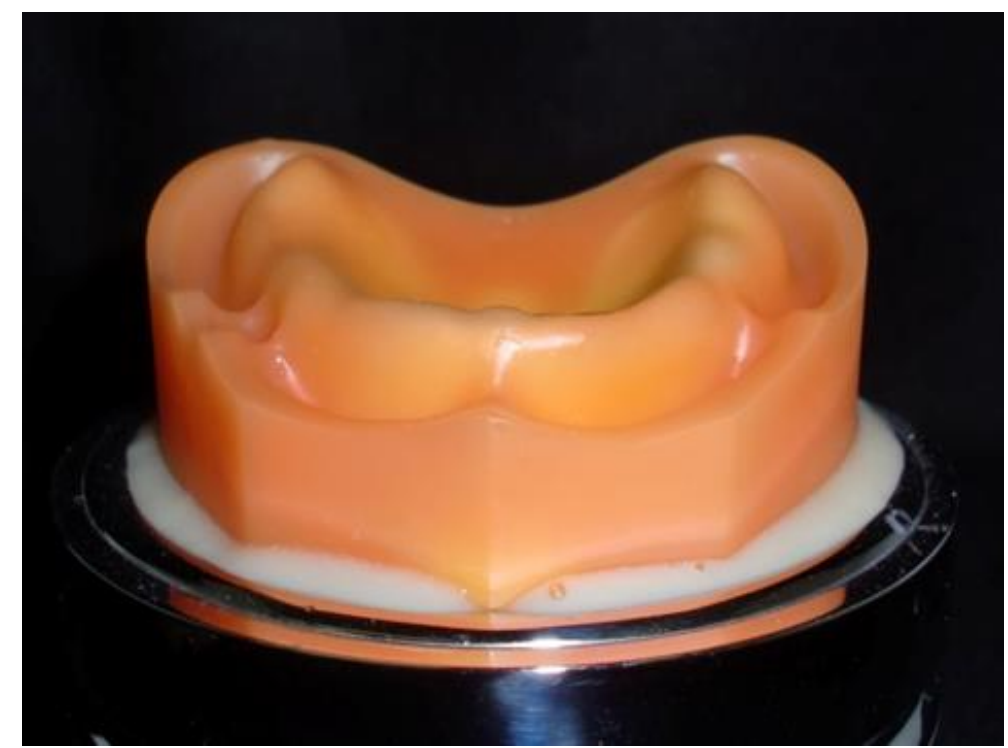

288

289

290

291

292

293

294

295

296

297

298 figure_3 0.9-mm traction ring made of Co-Cr was attached to the center of the palate 


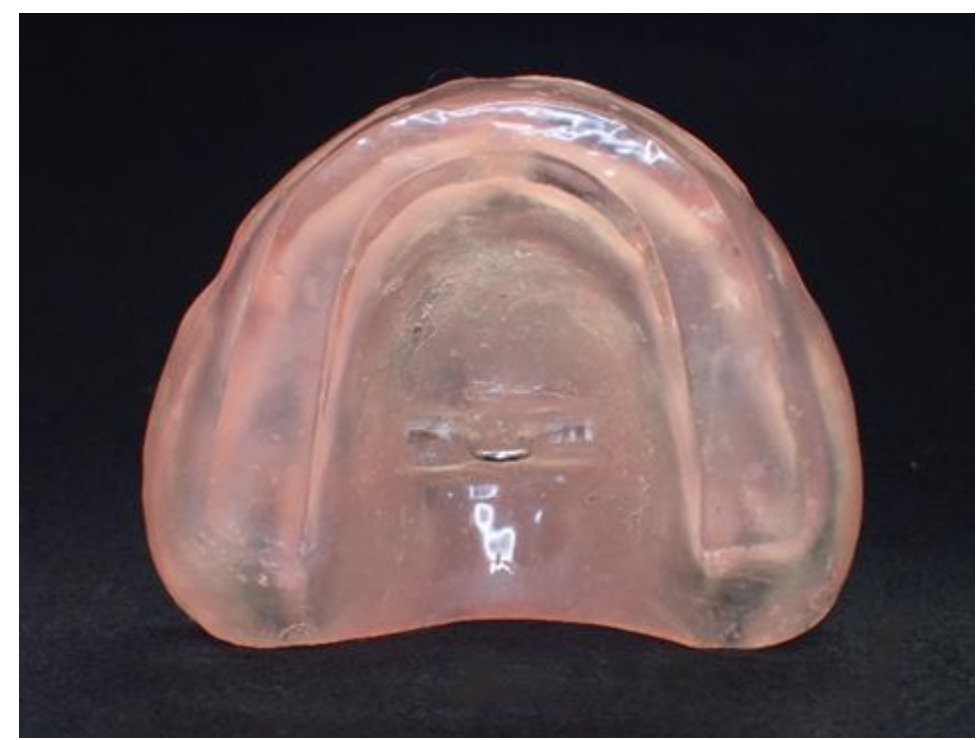

300

301

figure_4

A digital force gauge

(Digital Force

gauge RX Series ${ }^{\circledR}$;

302 AIKOHENGINEERING, Tokyo, Japan)

303

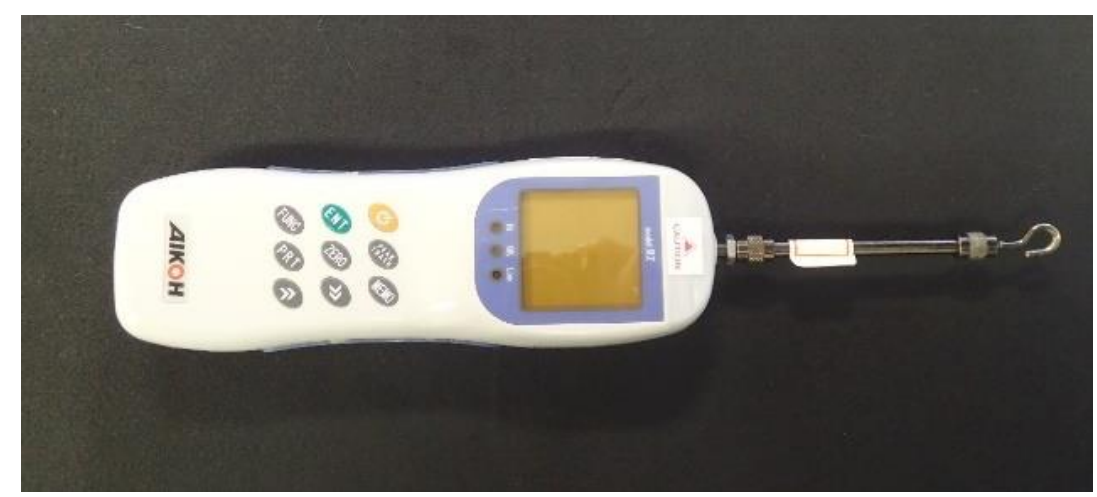

304

305

306

307

308

309

310

311

312

313

314

315

316 figure_5 Resting retention experiment 


\section{Loaded $\rightarrow$ pulled}

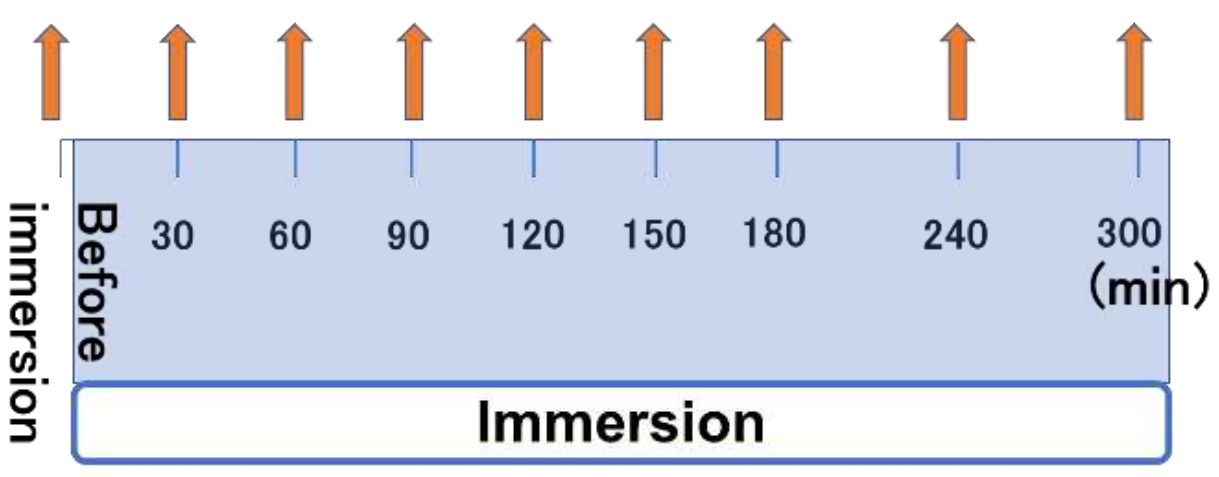

319

figure_6 Experiment of retention during function

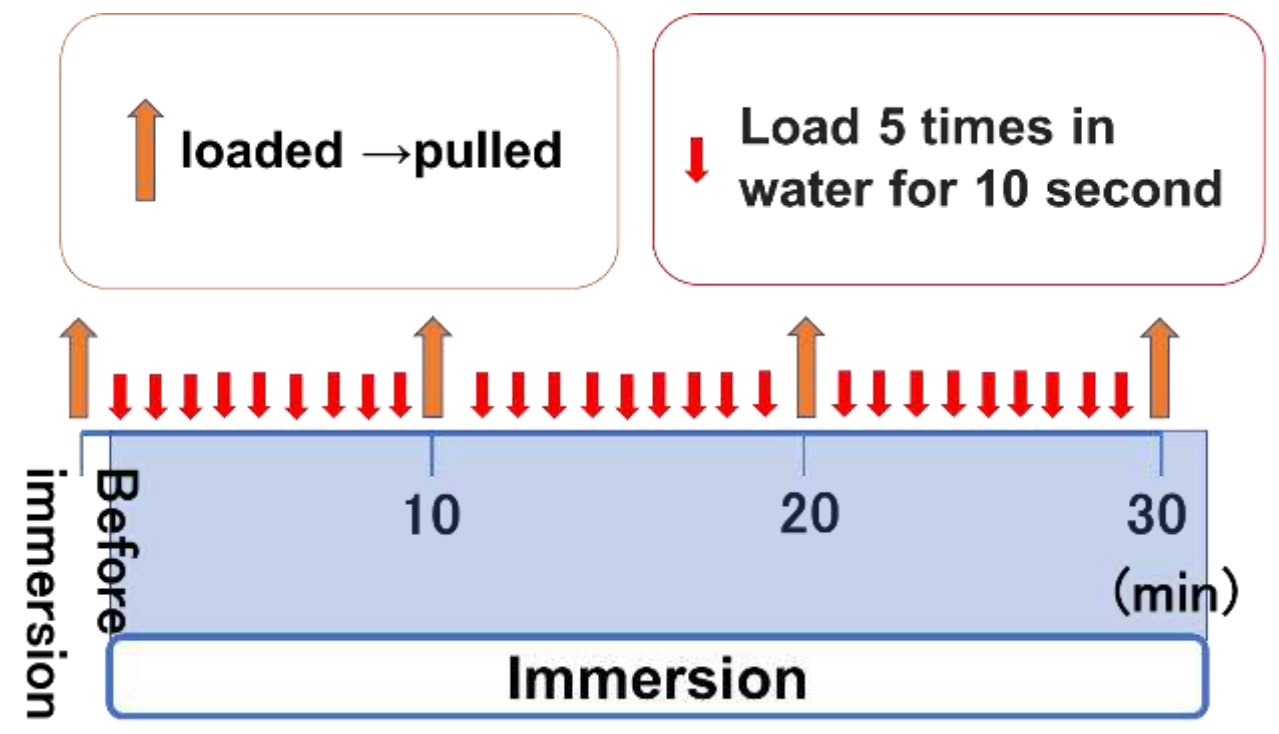



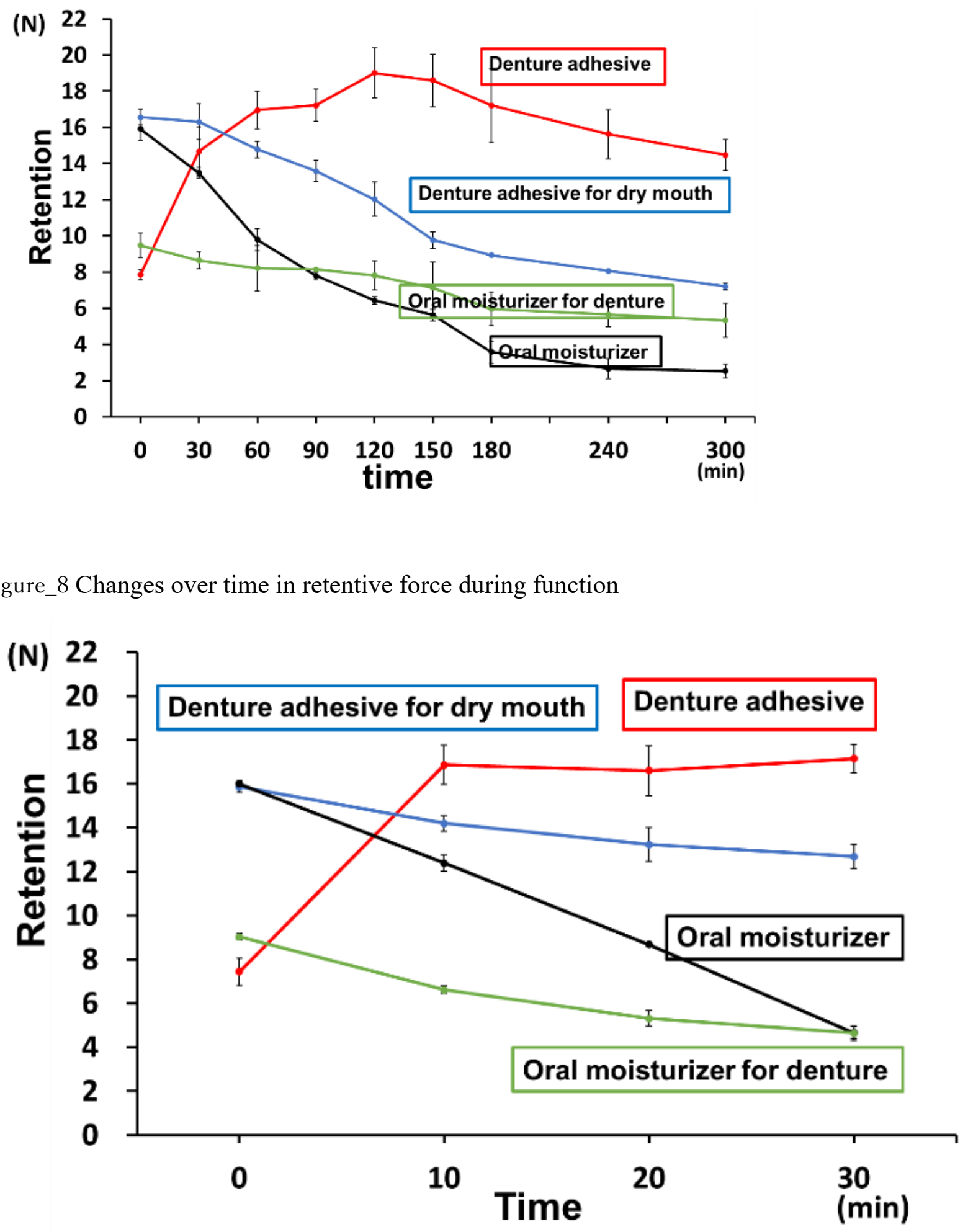
338 figure_9 Comparison of changes over time at rest and during function

339

\section{Changes at rest}

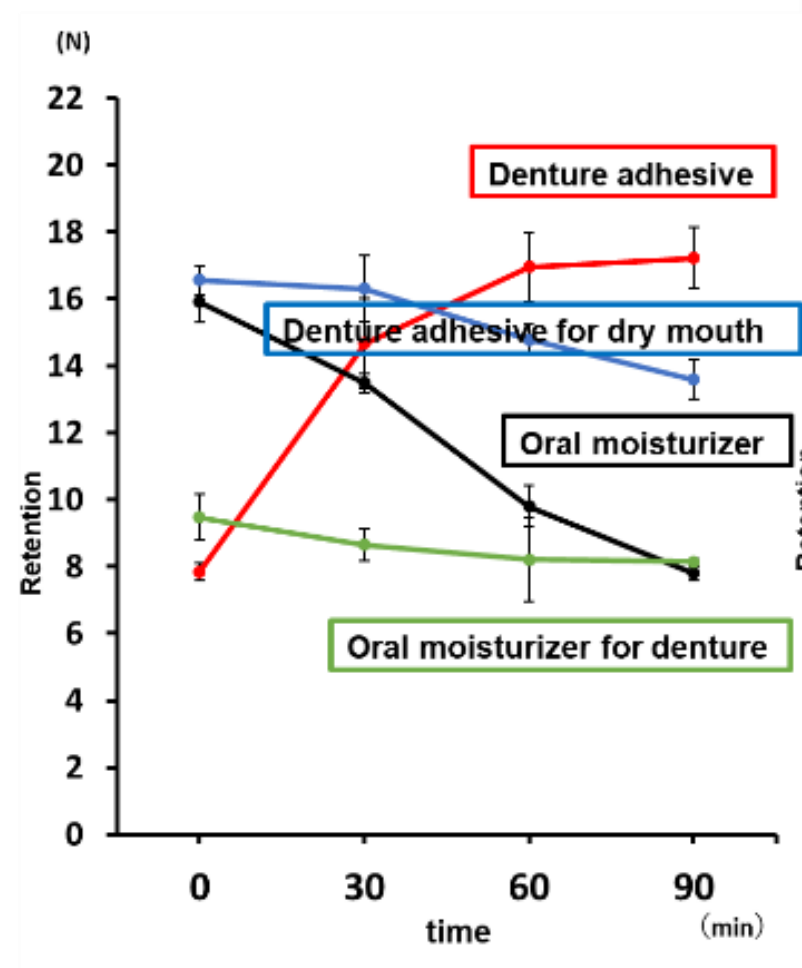

Changes during function

(N)

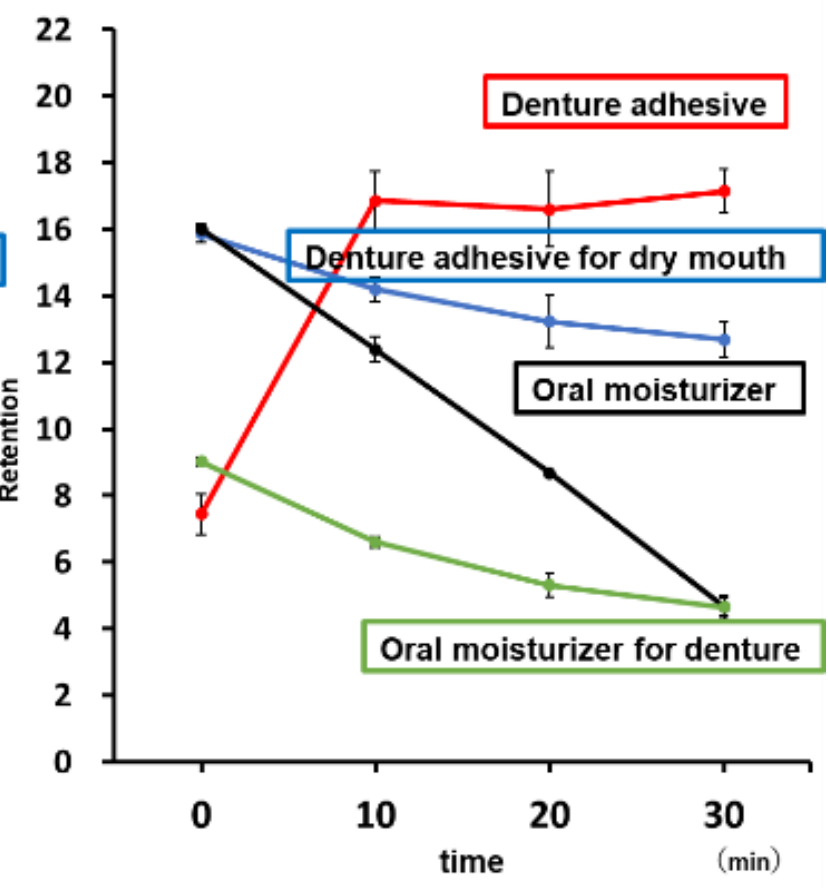

340

References

1. Cabinet Office. Annual Report on the Aging Society. 2018 (Summary). Available from:

https://www8.cao.go.jp/kourei/english/annualreport/2018/2018pdf_e.html [Accessed: ]. 
349 of dentition status on physical disability, mental impairment, and mortality in

350 institutionalized elderly people. J Dent Res. 2001;80:340-5.

3. Triantos D. Intra-oral findings and general health conditions among institutionalized

353 and non-institutionalized elderly in Greece. J Oral Pathol Med. 2005;34:577-82.

4. Panek H, Krawczykowska H, Dobosz A, Napadek P, Panek BA, Sosna-Gramza M.

Follow-up visits as a measure of adaptation process to removable prostheses.

Gerodontology. 2006;23:87-92.

358

5. Polzer I, Schimmel M, Mller F, Biffar R. Edentulism as part of the general health problems of elderly adults. Int Dent J. 2010; 60: 143-55.

6. Navazesh M, Brightman VJ, Pogoda JM. Relationship of medical status, and salivary flow rates in adults of different ages. Oral Surg Oral Med oral Pathol Oral Radiol Endod. 1996;81:172-6. 
367 symptoms and function in removable denture wearers in Japan. Oral Surg Oral Med oral

368 Pathol Oral Radiol Endod. 2005;99:704-10.

369

370 8. Jacobson TE, Krol AJ. A cotemporary review of the factors involved in complete

371 denture retention, stability, and support. Part I: Retention. J Prosthet Dent 1983;49:5-15.

372

373 9. Oliveira Junior NM, Rodriguez LS, Marin DOM, Paleari AG, Pero AC, Compagnoni

374 MA. Masticatory performance of Conplete denture wearers after using two adhesives: A

375 crossover randomized clinical trial. J Prosthet Dent. 2014;112:1182-7.

376

377

10. Adisman, IK. The use of denture adhesives as an aid to denture treatment. J Prosthet

378

Dent. 1989;62:711-5.

379

380 11. Chen F, Mao T, Cheng X. pH and effects on Streptococcus mutans growth of denture

381 adhesives: an in vitro study. Gerodontology. 2012;31:95-100.

382

383

12. Harada-Hada K, Hong G, Abekura H, Murata H. Evaluation of the efficiency of

384 denture cleaners for removing denture adhesives. Gerodontology. 2016;33(4):453-60. 
386 13. Yamagaki K, Kitagawa N, Sato Y, Okane M, Mashimo J. The relationship between

387 the physical properties of oral moisturizer and denture retention force. Jpn J Gerodont. $2011 ; 26: 402-11$.

389

390

14. Aoyagi K, Sato Y, Kitagawa N, Okane M, Kakuda T, Takayama M. Development of

a simple chair-side evaluation method for complete denture retention forces and its

reproducibility. Jpn J Gerodont. 2014;29:21-8.

393

394

15. Tsubakida K, Sato Y, Kitagawa N, Nakatsu M, Kana T, Takuya K, Mari T, Masae I.

395

Factors affecting the selection of denture adhesive or BT by wearers of maxillary

complete dentures. JSM Dent. 2017;5:1099.

16. Ohno T, Itatsu R, Moriya M, Sato Y, Sumi Y. Evaluation of the physical properties of a newly developed denture adhesive for patients with dry mouth. J Prosthodont Res. 2019;63:157-61. 
403 T. The effect of polyacrylic acid natrium for adhesive agent of maxillary prosthesis and 404 unstable plate denture. Oral Ther Pharmacol 1985;4:41-9.

406 18. Kakuda T, Sato Y, Kitagawa N, Nakatsu M, Aoyagi K, Takayama M, Tsubakida K.

407 Examination of Optimal Sites and Loading Methods for Measuring Maxillary Complete 408 Denture Retention. Jpn J Gerodont. 2015;30:25-36.

409

410 
Figures

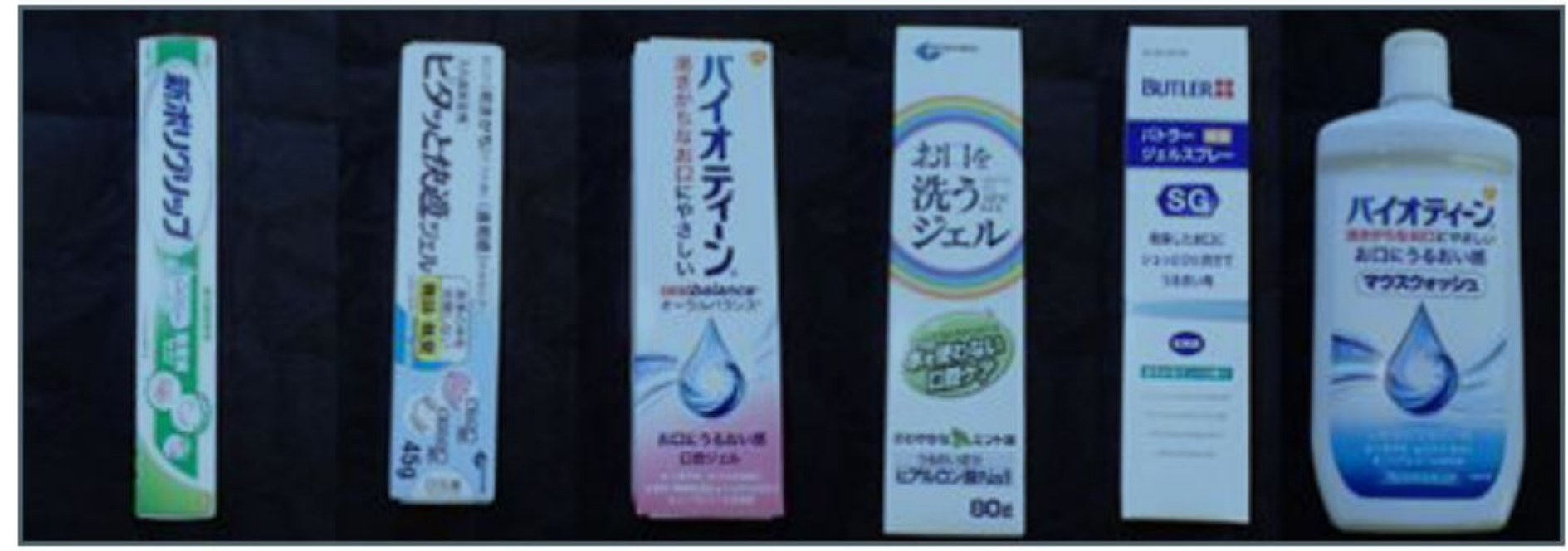

Figure 1

Test Samples

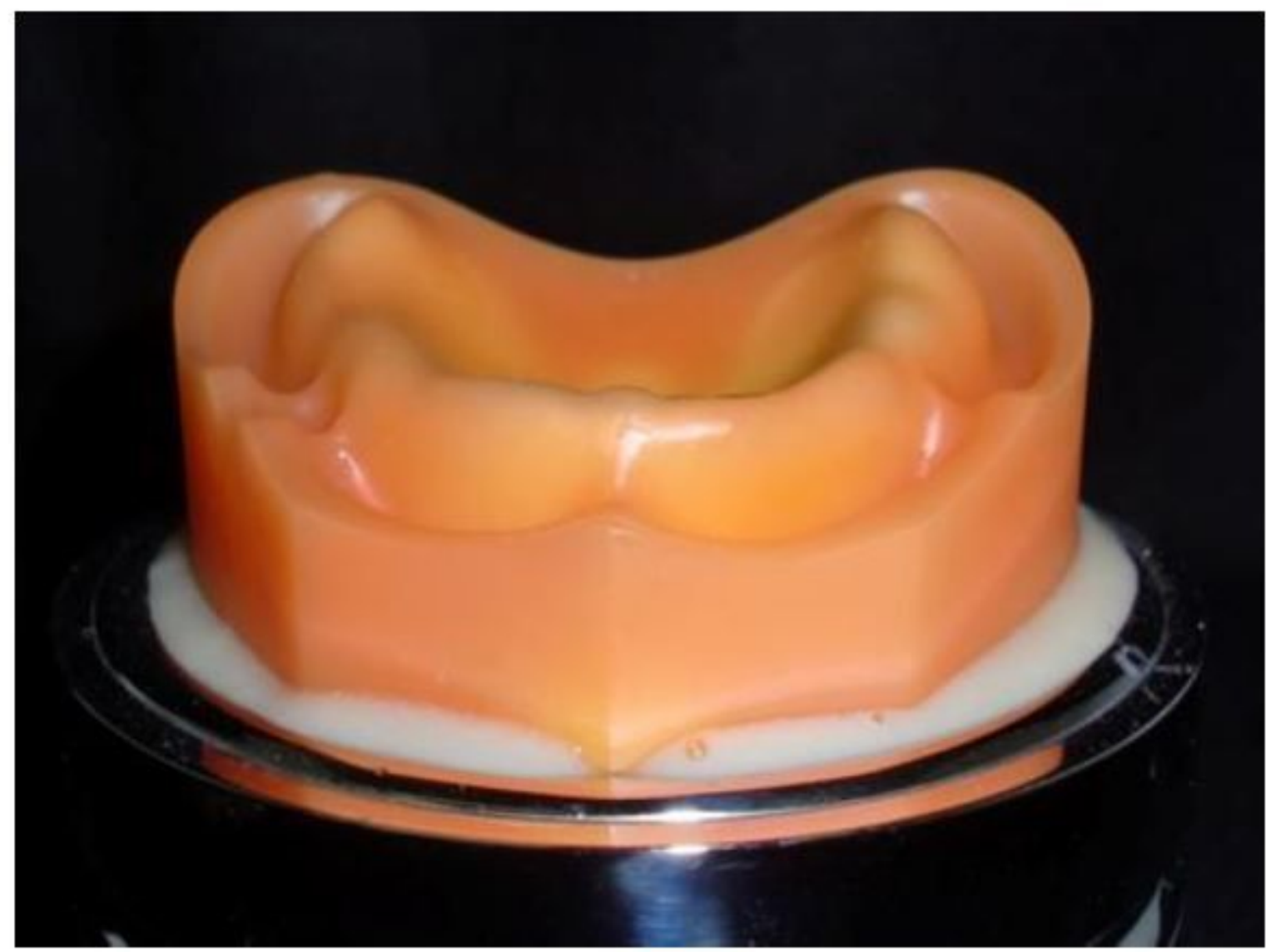

Figure 2 
silicone edentulous jaw ridge model

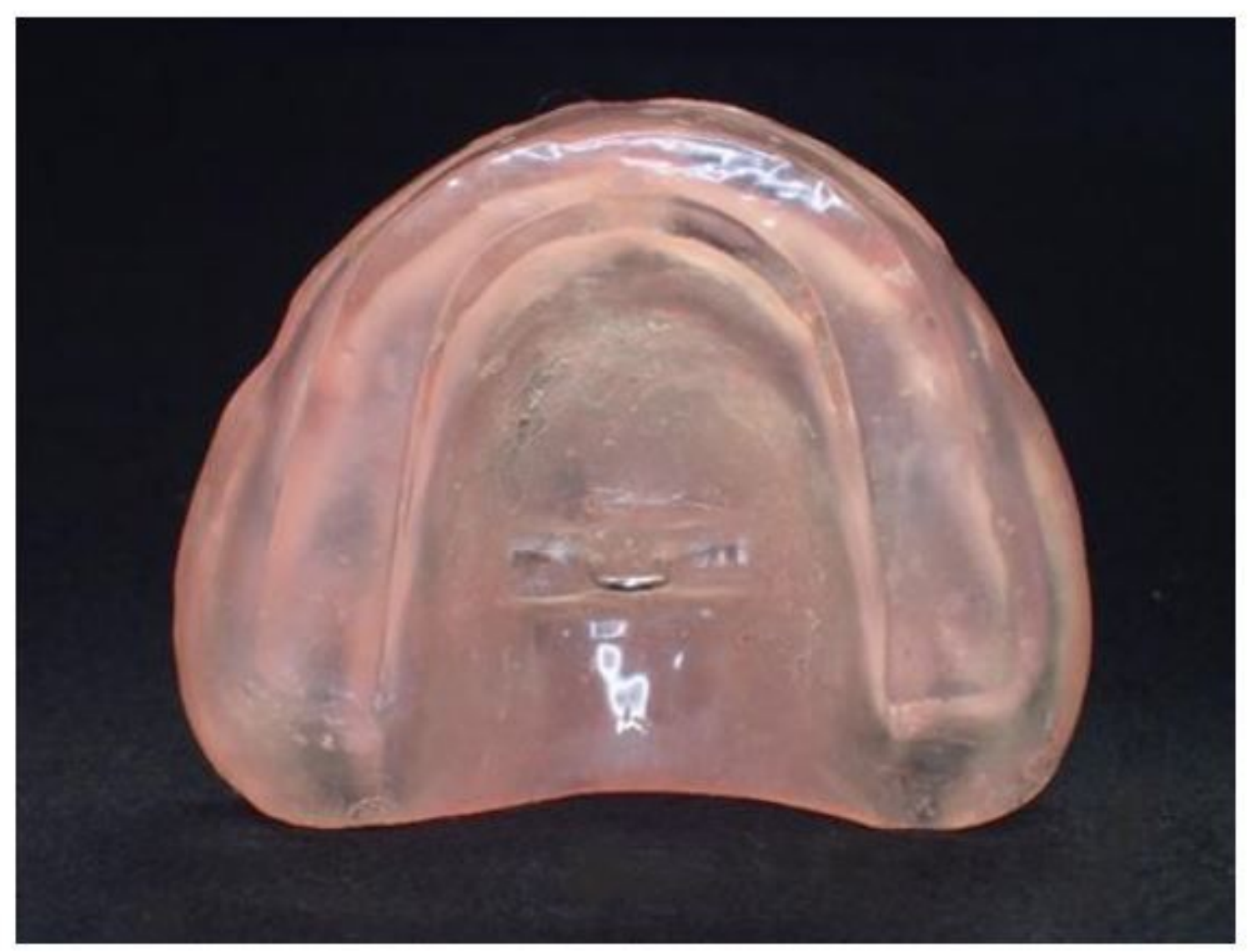

\section{Figure 3}

0.9-mm traction ring made of Co-Cr was attached to the center of the palate

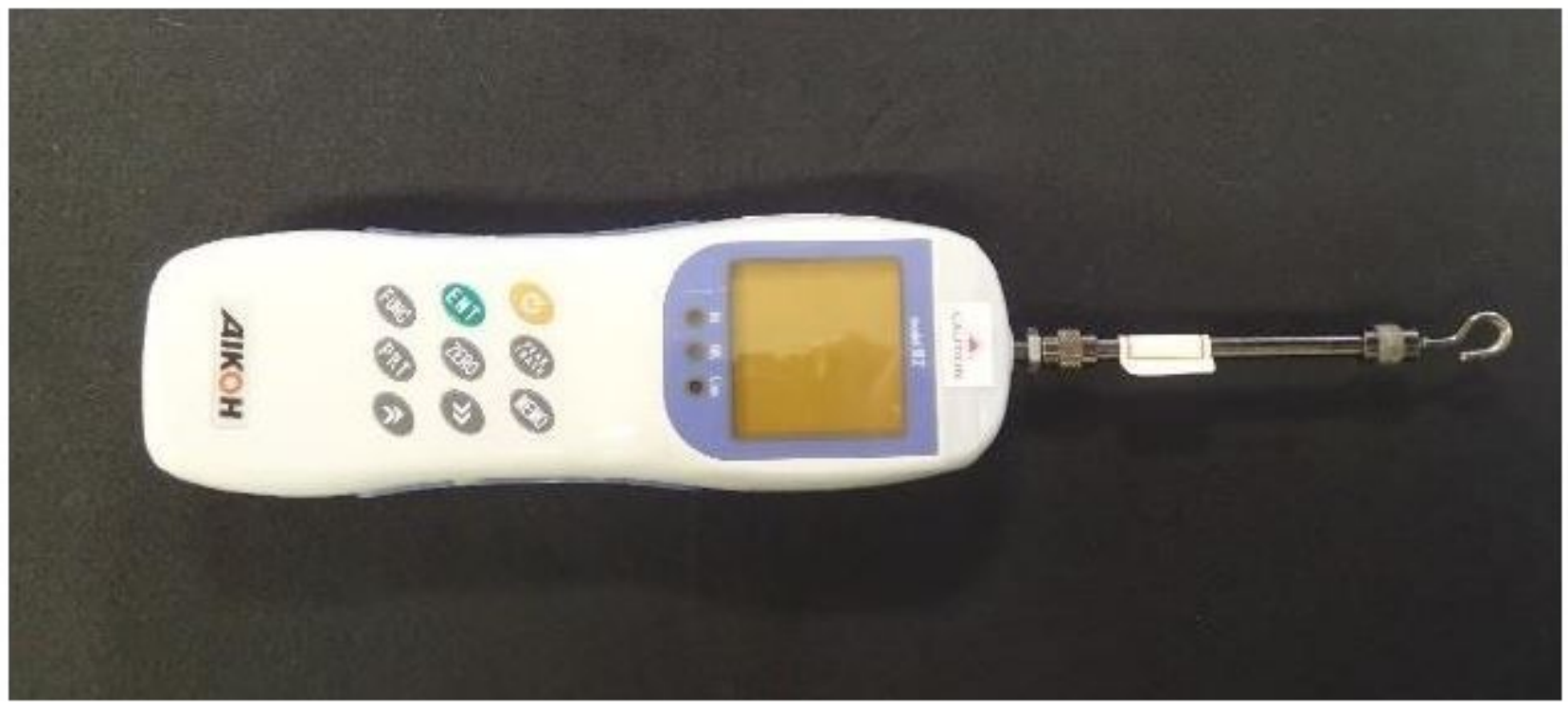

Figure 4 


\section{Loaded $\rightarrow$ pulled}
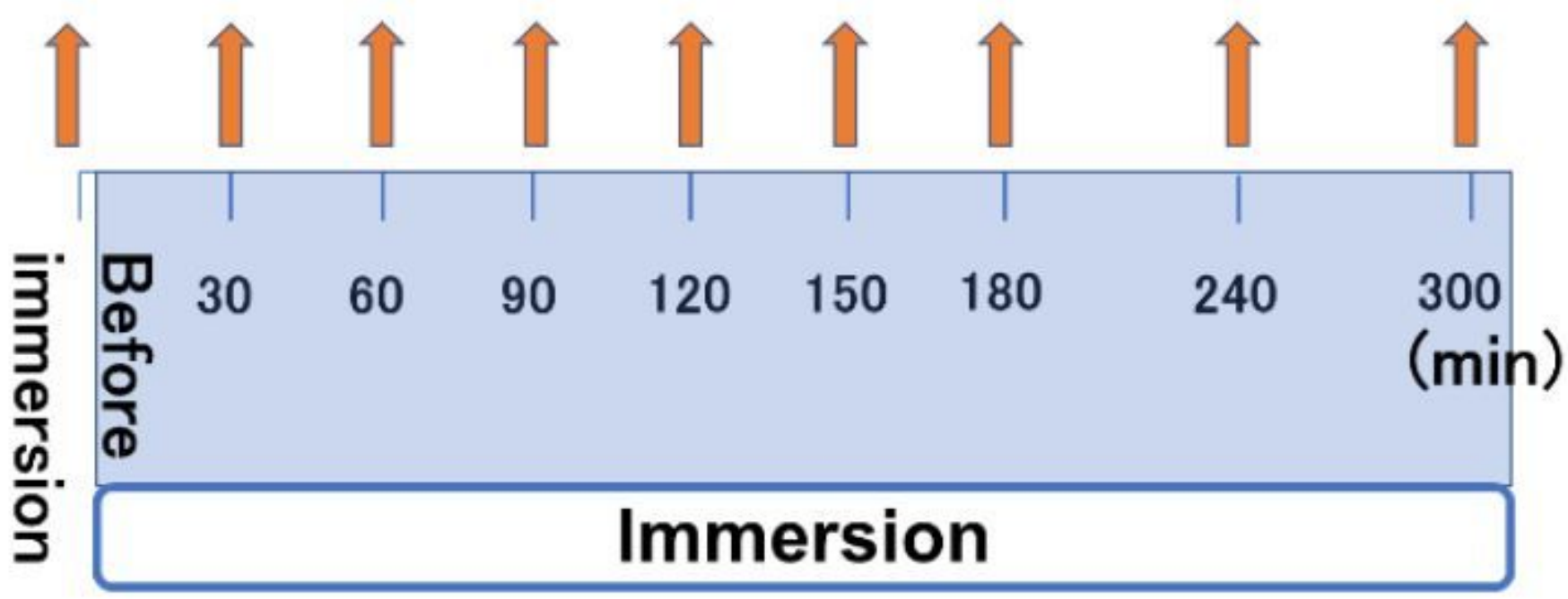

Figure 5

Resting retention experiment 


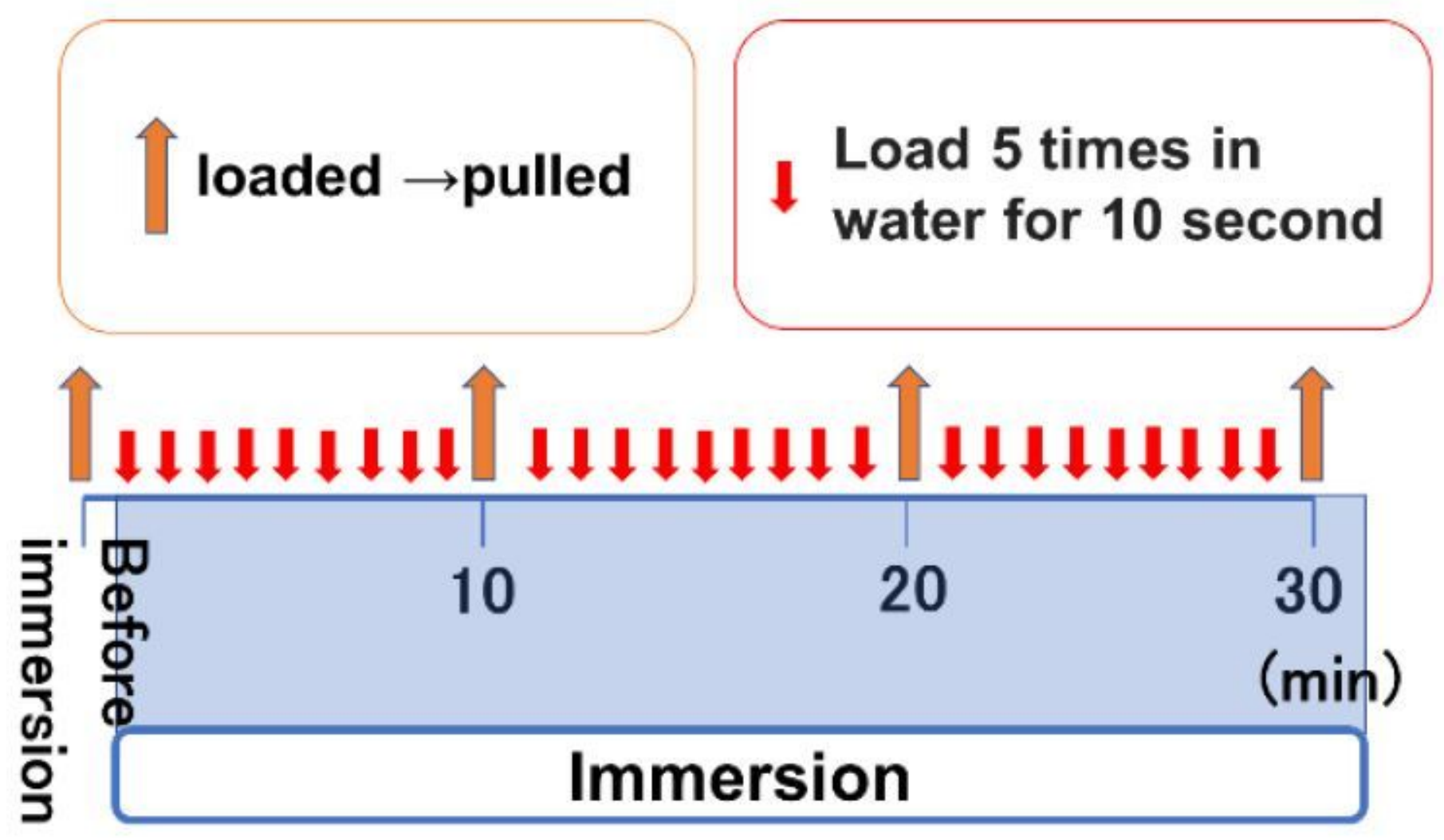

Figure 6

Experiment of retention during function 


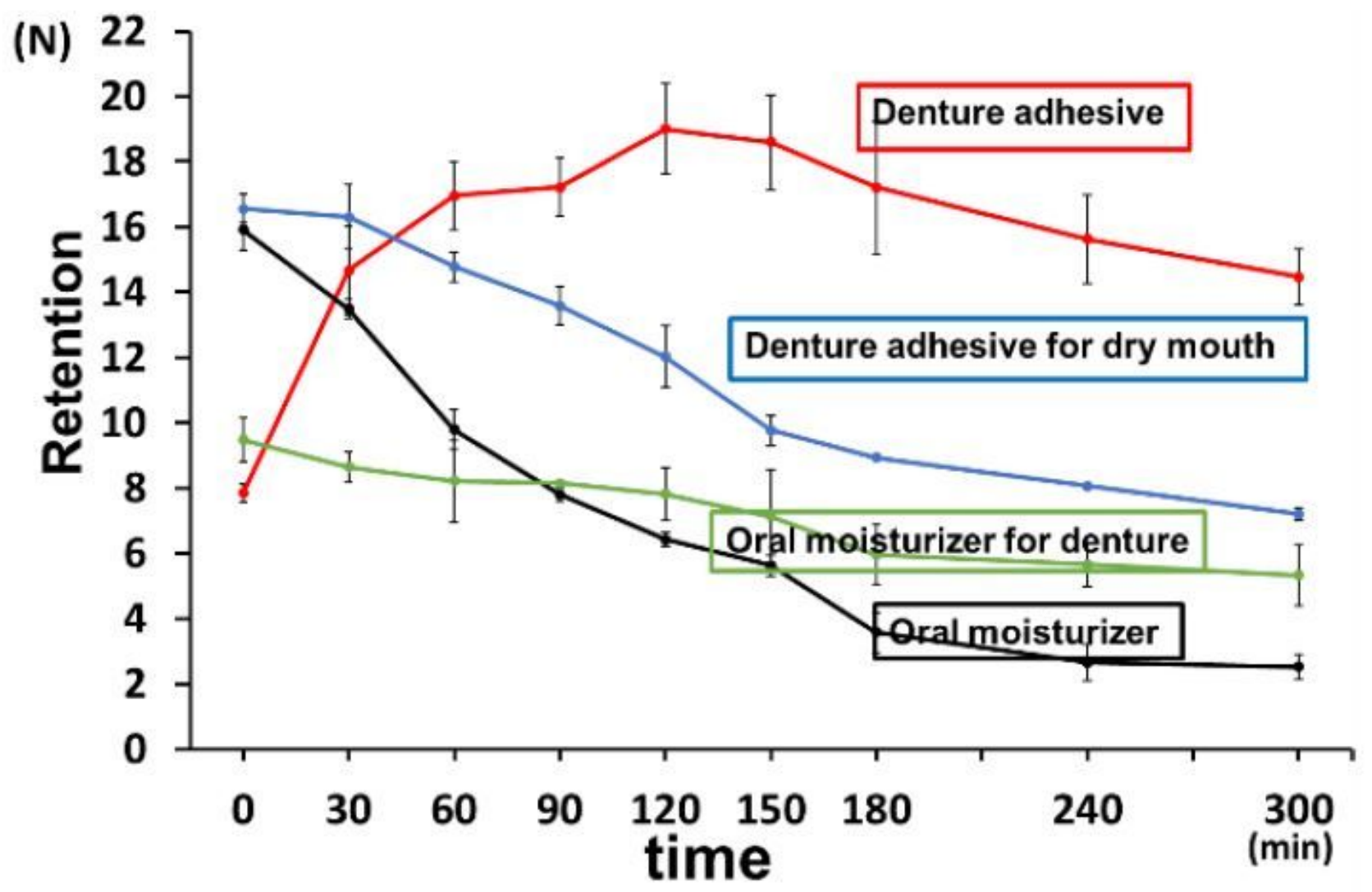

Figure 7

Change over time in the retentive force at rest 


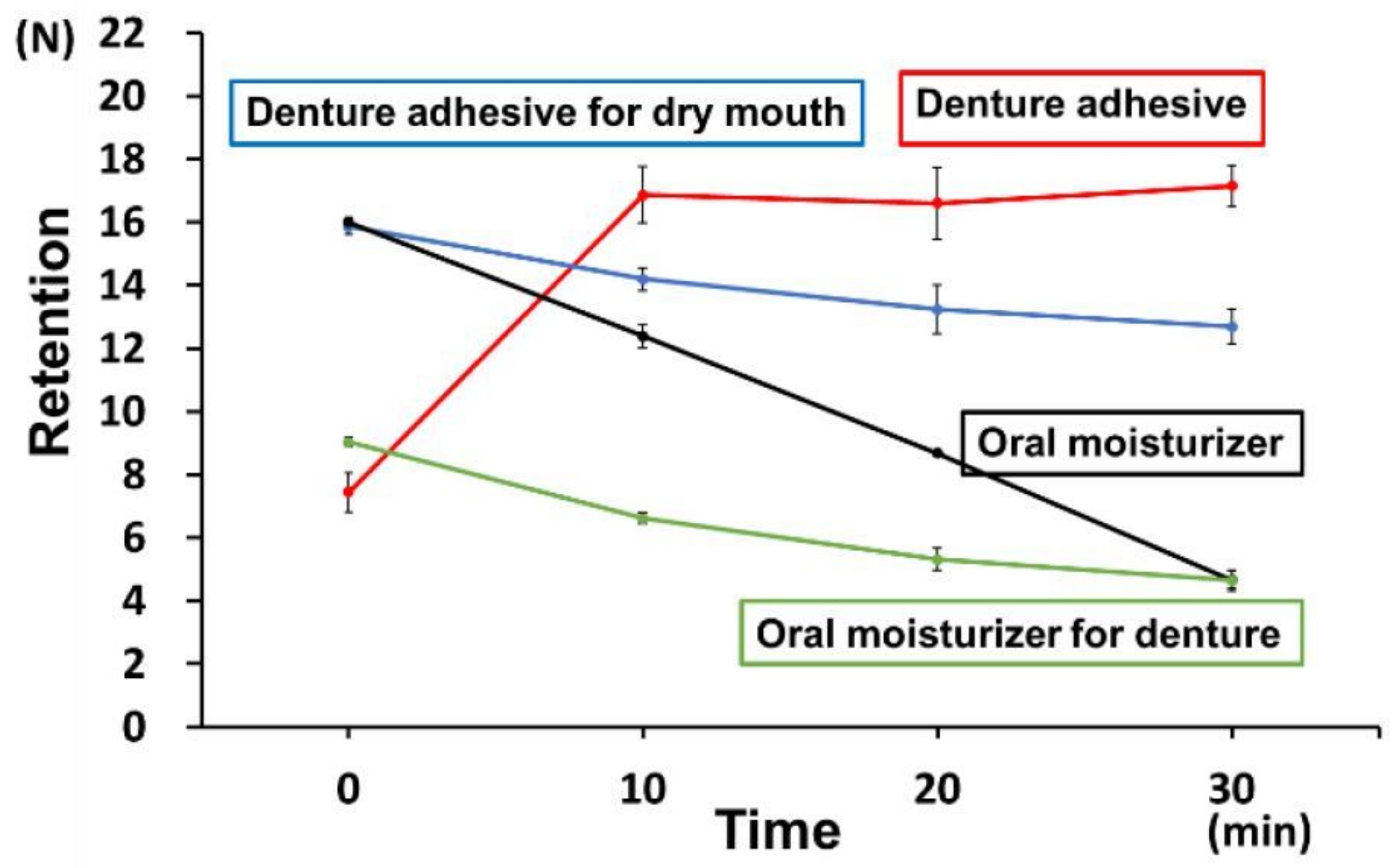

Figure 8

Changes over time in retentive force during function 


\section{Changes at rest}

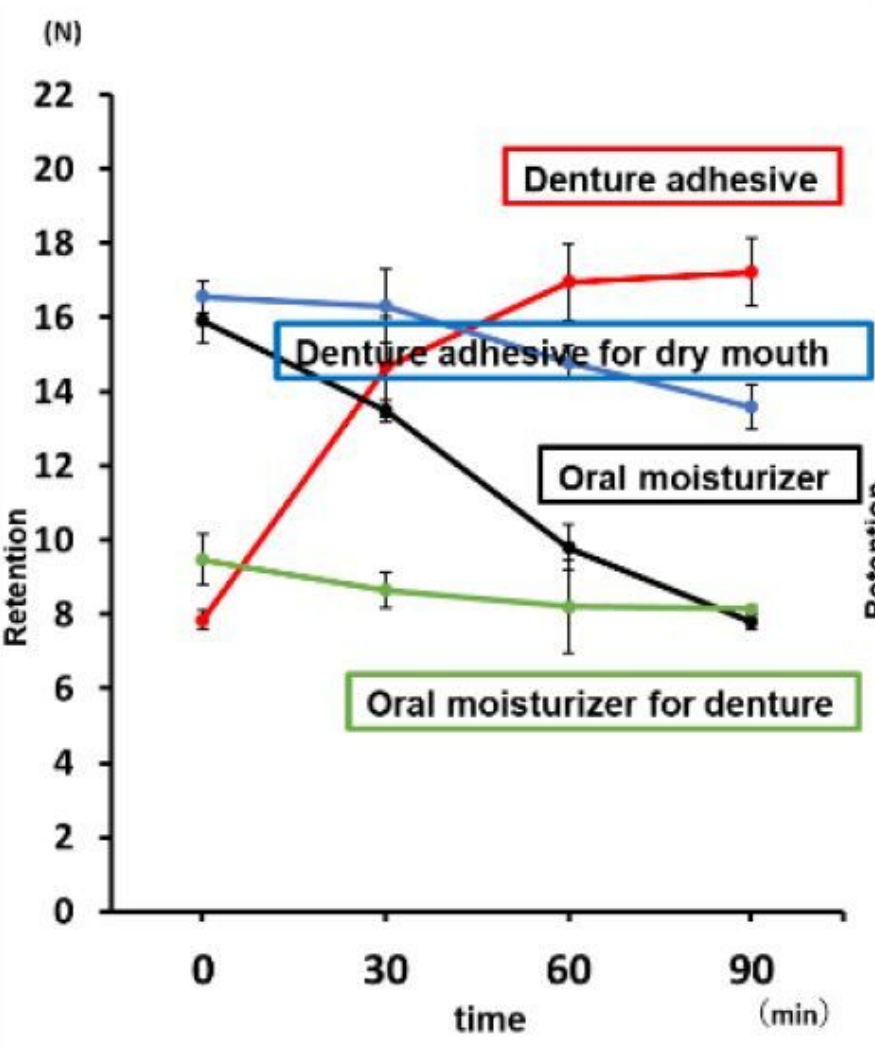

Changes during function

(N)

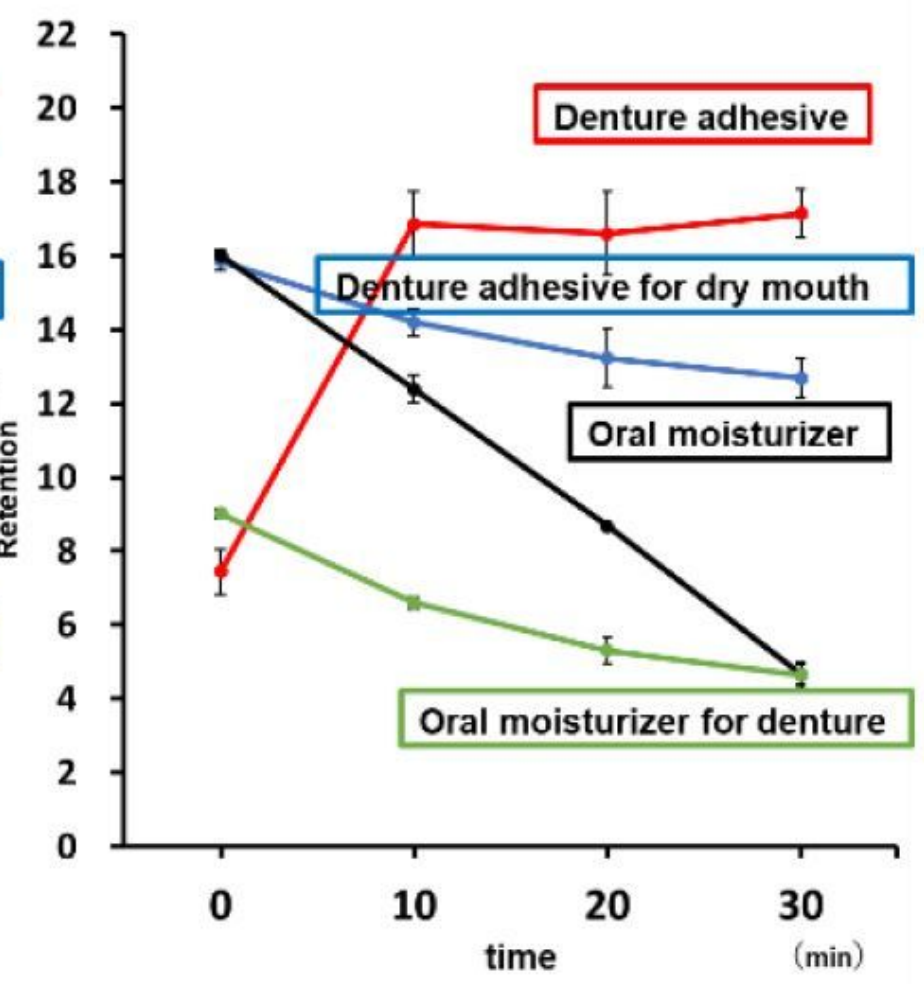

Figure 9

Comparison of changes over time at rest and during function 